Recibido: 02/03/2021 --- Aceptado: 29/03/2021 --- Publicado: 12/04/2021

\title{
MENOS NO SIEMPRE ES MÁS. CÓMO EL TAMAÑO DE LOS MEDIOS CONDICIONA LAS PRÁCTICAS EXTRACURRICULARES EN PERIODISMO
}

\section{LESS IS NOT ALWAYS MORE. HOW MEDIA SIZE CONDITIONS INTERNSHIPS IN JOURNALISM}

8 Manuel García-Borrego: Universidad de Málaga. España. manoletus@uma.es

Bernardo Gómez-Calderón²: Universidad de Málaga. España. bjgomez@uma.es

817 Alba Córdoba-Cabús $3:$ Universidad de Málaga. España. albacordoba@uma.es

\section{Cómo citar el artículo:}

García-Borrego, M., Gómez-Calderón, B. y Córdoba-Cabús, A. (2021). Menos no siempre es más. Cómo el tamaño de los medios condiciona las prácticas extracurriculares en Periodismo. Revista de Comunicación de la SEECI, 54, 79-95. http://doi.org/10.15198/seeci.2021.54.e634

\section{RESUMEN}

El objetivo de esta investigación es examinar las condiciones en que desarrollan las prácticas extracurriculares los estudiantes de Periodismo en función del tamaño de la empresa en que realizan su estancia, distinguiendo entre las pequeñas (de 1 a 20 empleados), medianas (más de 20 y menos de 100) y grandes (100 o más). Para ello se suministró un cuestionario a estudiantes de las universidades de Málaga y Complutense de Madrid que habían realizado prácticas en medios durante el curso 2017-2018 $(n=144)$. Los resultados obtenidos evidencian la existencia de patrones

\footnotetext{
${ }^{1}$ Manuel García-Borrego: Personal Investigador en Formación con beca FPU en la Universidad de Málaga, ha publicado artículos sobre la formación en Periodismo y las prácticas externas en revistas como El Profesional de la Información, Latina o Estudios sobre el Mensaje Periodístico. manoletus@uma.es

2 Bernardo Gómez-Calderón: Profesor Titular de Universidad. Imparte docencia desde 1998 y cuenta con más de 60 publicaciones científicas entre artículos, libros y capítulos de libro. Investigador de manera ininterrumpida en proyectos nacionales y autonómicos desde 2001. bjgomez@uma.es

${ }^{3}$ Alba Córdoba-Cabús: Personal Investigador en Formación (FPU) en la Universidad de Málaga. Graduada en Periodismo y Máster en Investigación sobre Medios de Comunicación, Audiencias y Práctica Profesional en Europa. Sus principales trabajos versan sobre Periodismo de Datos. albacordoba@uma.es
} 
García-Borrego, M., Gómez-Calderón, B. y Córdoba-Cabús, A Menos no siempre es más.

de prácticas distintos: en las empresas pequeñas la retribución tiende a ser mayor aunque es también donde en mayor medida se dan casos de prácticas no remuneradas-, los estudiantes dedican de media menos tiempo que sus compañeros, su criterio es más tenido en cuenta, la relación con los tutores es más fluida y suelen ocupar un área estable. En las grandes se produce el fenómeno opuesto, y es sin embargo donde existe un mayor sentimiento de pertenencia por parte de los alumnos - la mayoría querría continuar haciendo carrera en ellas-, mientras que en los pequeñas y las medianas prima la preferencia por el cambio de aires. En conjunto, la investigación muestra perfiles de prácticas bien diferenciados en base a la variable seleccionada, lo cual invita a una reflexión sobre las características de los centros de acogida y su papel en el currículo formativo del alumnado de Periodismo.

PALABRAS CLAVE: Prácticas profesionales - Periodismo - Rutinas periodísticas Condiciones laborales - Becarios - Formación universitaria - Medios de comunicación

\section{ABSTRACT}

The aim of this research is to examine how the conditions of the media internships in Journalism change according to the size of the company, distinguishing between small ( 1 to 20 employees), medium (more than 20 and fewer than 100) and large ones (100 or more). In order to achieve this, a questionnaire was provided to those students from University of Málaga and Complutense University of Madrid who had carried out their internships in media outlets during the 2017-2018 academic year $(n=144)$. The results indicate the existence of different internship patterns: in small companies the retribution tends to be higher -although it is also where more internships remain unpaid-, students dedicate less time than their peers, their opinions are more heard, the relationship with their tutors is more fluid and they usually stay in a stable area of the company. In big companies the opposite phenomenon emerges, and it is nevertheless where there is a greater sense of belonging by the students -the majority of them would like to continue making a career in them-, while in small and medium companies the preference for a change prevails. In summary, the research shows well-differentiated internship profiles based on the size variable, which invites reflection on the characteristics of the companies and their role in the formative curriculum of Journalism students.

KEY WORDS: Media internships - Journalism - Professional routines - Working conditions - Trainees - University training - Media companies

\section{MENOS NEM SEMPRE É MAIS. COMO O TAMANHO DOS MEIOS DE COMUNICAÇÃO CONDICIONA AS PRÁTICAS EXTRACURRICULARES NO JORNALISMO RESUMO:}

O objetivo desta pesquisa é analisar as condições nas que se desenvolvem as práticas extracurriculares dos alunos de jornalismo em função do tamanho da 
García-Borrego, M., Gómez-Calderón, B. y Córdoba-Cabús, A Menos no siempre es más. Cómo el tamaño de los medios condiciona las prácticas extracurriculares en Periodismo

empresa em que trabalham, diferenciando entre as pequenas (de 1 a 20 empregados), médias (mais de 20 e menos de 100) e grandes (100 ou mais). Para isto se usou um questionário a alunos das universidades de Málaga e Complutense de Madrid que tinham feito práticas em meios de comunicação durante o curso 2017-2018 ( $n=144)$. Os resultados obtidos evidenciam existência de padrões de práticas diferentes: nas empresas pequenas a retribuição tem uma tendência de ser maior - mesmo que também é onde se dão casos de práticas não remuneradas -, os alunos dedicam em média menos tempo que seus colegas, o critério é mais levado em consideração, a relação com os tutores é mais fluida e geralmente ocupam uma área estável. Nas grandes empresas se produz o fenômeno oposto, mas é também onde existe um maior sentimento de pertencimento por parte dos alunos -a maioria pretende continuar fazendo parte delas - , enquanto nas pequenas e nas medianas é maior a preferência pela mudança de ambiente. Em conjunto, a pesquisa mostra perfis de práticas bem diferenciados dependendo da variável selecionada, o que convida a uma reflexão sobre as características dos centros de acolhida e seu papel no currículo formativo do aluno de jornalismo.

PALAVRAS CHAVE: Práticas profissionais - Jornalismo - Rotinas jornalísticas Condições laborais - Bolsistas - Formação universitária - Meios de comunicação

\section{INTRODUCCIÓN}

Las prácticas externas auspiciadas por la universidad cumplen una doble función para los alumnos: por un lado, los acercan a la realidad laboral para tratar de conseguir una "formación integral (...) a través de programas de cooperación educativa", siendo este el objetivo para el que fueron inicialmente concebidas (véase Real Decreto 592/2014, de 11 de julio); y por otro, suponen para ellos la principal vía de entrada a la profesión periodística (APM, 2010-2018). Es decir: lo que en su origen poseía una naturaleza eminentemente formativa representa en el momento actual una pasarela necesaria hacia un futuro puesto de trabajo, por lo que el componente académico de esta actividad ha acabado conviviendo de hecho con el puramente laboral.

Al desarrollar sus prácticas, los estudiantes acceden a una comunidad profesional en la que aprenden - de manera tácita y a través de una praxis continuada - tanto del resto de redactores como de las situaciones a las que deben enfrentarse en su quehacer diario (Dreyfus y Dreyfus, 1986; Lave y Wenger 1991; Splichal y Sparks, 1994; Wenger, 1998; Gravengaard y Rimestad, 2014). Este aprendizaje de las rutinas del periodismo, que abarca desde los sistemas de valores hasta las pautas de trabajo, resulta clave en la socialización del alumno y en su posterior desarrollo profesional (Hall, 1976; Cohen-Scali, 2003; Cotter, 2010).

A pesar de la relevancia de esta primera toma de contacto con el ejercicio del periodismo en la configuración de la cultura laboral de los futuros redactores, las condiciones en las que se desarrollan estas prácticas y sus posibles efectos, así como 
García-Borrego, M., Gómez-Calderón, B. y Córdoba-Cabús, A Menos no siempre es más.

Cómo el tamaño de los medios condiciona las prácticas extracurriculares en Periodismo

las circunstancias que favorecen un mayor aprovechamiento de las mismas, apenas han sido abordados hasta ahora por la academia.

En España, los primeros estudios arrancan con Cantalapiedra, Coca y Bezunartea (2000), que detectaron en el País Vasco una de las disfunciones más comunes de este tipo de estancias: a pesar de estar aún en fase de formación, una vez que los estudiantes encuestados llegaban a las redacciones de medios realizaban las tareas propias de un profesional de plantilla. Los medios, en muchos casos con más becarios que redactores sénior, dependían de los alumnos para su subsistencia, por lo que la necesidad de buscar la máxima productividad terminaba por imponerse a la vertiente formativa de las prácticas.

Años más tarde, Blanco (2005) desvelaba una realidad prácticamente idéntica en Málaga: casi nueve de cada diez estudiantes de prácticas consideraban que su trabajo suplía al de un contratado. Eran mayoría quienes afirmaban superar amplia y sistemáticamente los horarios firmados por convenio y quienes sentían que las instituciones de acogida abusaban laboralmente de ellos. Una tercera parte de los consultados, de hecho, no recibía compensación económica alguna por sus tareas. Aun así, el $97,5 \%$ percibía las prácticas como un buen complemento para su formación, y la satisfacción global era muy alta.

Lamuedra (2007), por su parte, analizó las prácticas curriculares de la Universidad Carlos III de Madrid, donde, de nuevo, se producía una perversión del sistema en tanto que a los estudiantes se les exigía mayor dedicación horaria de la estipulada para llenar el hueco que debía ocupar un trabajador de plantilla, en el marco de unas prácticas incorporadas como asignatura en el plan de estudios $-\mathrm{y}$, por consiguiente, no remuneradas-. Los alumnos, lejos de lamentar esta situación, se mostraban satisfechos de poder experimentar de primera mano, tal y como lo haría un redactor sénior, las múltiples casuísticas del ejercicio de la profesión -incluso aquellas que incurrían en una mala praxis periodística-.

Paralelamente a estas publicaciones emergieron voces que abogaban por una mayor regulación de las prácticas para marcar distancias claras entre su finalidad formativa y los intereses empresariales, cuyo maridaje no siempre resulta compatible (Udías, 2006). Desde entonces transcurrió una década hasta que volvió a realizarse un trabajo de campo para medir los avances en el desarrollo de las prácticas; antes, Pérez-Serrano, Rodríguez-Barba y Rodríguez-Pallarés (2015) habían analizado las ofertas del servicio de prácticas de la Universidad Complutense de Madrid, concluyendo que la remuneración media se situaba en torno a los 225 euros por cinco horas al día, aunque existían casos que aportaban unos exiguos 175 euros por casi 35 horas semanales.

En los últimos años, pues, se ha retomado el estudio empírico de las prácticas externas a través de cuestionarios a los alumnos. En Málaga, García-Borrego y Roses (2016) y García-Borrego, Roses y Farias (2017) observaron que la llamada "bolsa de ayuda" al estudiante había crecido ligeramente con respecto al trabajo anterior de Blanco (2005) -la mayoría se situaba en los 360 euros que estipula como mínimo la

Revista de Comunicación de la SEECI. 2021, n 54, 79-95 
García-Borrego, M., Gómez-Calderón, B. y Córdoba-Cabús, A Menos no siempre es más. Cómo el tamaño de los medios condiciona las prácticas extracurriculares en Periodismo

normativa de la universidad (UMA, 2020)-, aunque el tiempo dedicado seguía por encima de lo recogido en los convenios: 6,6 horas de media frente a las 5 regladas. En cualquier caso, la satisfacción global continuaba en niveles altos (7,64 puntos sobre 10), si bien una tercera parte de la muestra otorgaba una calificación de suspenso a las condiciones en que las prácticas se desarrollaban.

Estudios más recientes indican una nueva mejoría en las condiciones de trabajo de los estudiantes: en la UMA el horario apenas supera las 6 horas de media y se perciben unos 375 euros de remuneración, mientras que en la UCM, la jornada promedio desciende hasta las 5 horas y 45 minutos y la ayuda ronda los 270 euros mensuales, es decir, unos 45 más que en 2015 (Pérez-Serrano, Rodríguez-Barba y Rodríguez-Pallarés, 2015; Gómez-Calderón, García-Borrego y Fernández-Sande, 2019). En este último artículo se alerta asimismo sobre el alto porcentaje de estudiantes que realizan tareas con las que no están conformes por razones éticas casi la mitad de los encuestados-, así como la persistente desatención del tutor académico - nueve de cada diez alumnos de prácticas no traban contacto con él durante sus estancias-.

Todo esto difiere en buena medida de lo registrado en otros contextos nacionales como el danés, en que los estudiantes llevan a cabo funciones similares a los redactores de plantilla por 2.700 euros mensuales (Gravengaard y Rimestad, 2014), o el finés, donde la remuneración supera los 1.700 euros (Jaakkola, 2019). En Alemania, sin embargo, sí se registra una situación coincidente con la española, ya que los alumnos de Periodismo "trabajan un número largo de horas en turnos irregulares, a menudo por poco dinero, mientras tratan de construirse una reputación" (Gollmitzer, 2014: 834). La comparativa se complica al ampliar el foco a otros países, caso de los anglosajones, que no disponen de convenios de prácticas homologados en todo el territorio nacional (Willig, 2016).

\section{OBJETIVOS}

Partiendo de todo lo anterior, esta investigación hace por primera vez hincapié en el rol de la estructura redaccional de los medios - ya señalada como factor relevante en trabajos previos (Gómez-Calderón et al., 2019)- y persigue un objetivo fundamental: describir las similitudes y diferencias de las prácticas extracurriculares del grado en Periodismo en función del tamaño de la empresa de acogida.

\section{METODOLOGÍA}

La recogida de datos se hizo empleando la encuesta como herramienta metodológica, al estimarse que sería el mejor instrumento para recabar información sobre cada uno de los aspectos que determinan las prácticas. El cuestionario se elaboró a partir de las contribuciones de referencia de la literatura nacional e internacional, tanto las relacionadas expresamente con las estancias formativas en empresas como aquellas que abordan las rutinas profesionales y condiciones laborales de los periodistas sénior, entre las que figuran Splichal y Sparks (1994), Weaver (1998), APM (2005-2018), Túñez (2009), Weaver y Willnat (2012), Túñez y 
García-Borrego, M., Gómez-Calderón, B. y Córdoba-Cabús, A Menos no siempre es más. Cómo el tamaño de los medios condiciona las prácticas extracurriculares en Periodismo

Martínez-Solana (2014) o Suárez-Villegas (2015). Igualmente, se incluyó una batería de preguntas ad hoc para responder a variables hasta ahora no exploradas e íntimamente relacionadas con las prácticas como el contacto con los tutores. El cuestionario se estructuró finalmente en tres bloques: el primero recogía la información de registro de los encuestados (sexo, edad, sección, universidad de procedencia o remuneración), el segundo atendía a las rutinas profesionales (extensión de la jornada, número de piezas realizadas o frecuencia de la relación con los tutores) y el tercero se ocupaba de los niveles de satisfacción de los estudiantes (medidos a través de 11 variables: satisfacción los tutores, con la carga de trabajo, con la remuneración, etcétera).

La encuesta se puso en marcha en las universidades de Málaga y Complutense de Madrid, procurando aunar en una misma muestra dos perfiles de centros bien diferenciados: el que más alumnos acoge cada año (660 plazas de nuevo ingreso por curso), situado en la capital del país, y el de una provincia periférica con un numerus clausus más modesto (130). Al no ser posible, por razones de protección de datos, obtener las direcciones de correo de los estudiantes que habían realizado prácticas durante el curso 2017-2018, el cuestionario se envió por correo electrónico y en dos oleadas - los días 15 y 22 de octubre de 2018- a las tres últimas promociones del grado en Periodismo de ambas universidades (2013-2017, 2014-2018 y 2015-2019). De todos ellos respondieron, al cabo de un mes, 210 - un $29,2 \%$ del total de alumnos que habían hecho prácticas en ese período, según los datos proporcionados por los servicios de empleabilidad de cada institución-, que quedaron en 143 una vez discriminados quienes habían desempeñado tareas de comunicación institucional en gabinetes, al entender que, por ser estas empresas generalmente pequeñas y ocuparse de labores diferentes, distorsionarían la interpretación de los resultados.

Tras eliminar asimismo los archivos anómalos o erróneos con el programa estadístico SPSS, se realizó un análisis estadístico descriptivo, dividiendo la muestra en tres grupos en función del tamaño del medio en el que habían desarrollado sus prácticas: pequeño (de 20 empleados o menos), mediano (con más de 20 y menos de 100$)$ y grande (100 trabajadores o más).

\section{RESULTADOS}

\subsection{Descripción de la muestra}

La proporción de estudiantes en empresas pequeñas $(45,8 \%)$ era mayor que en medianas $(29,9 \%)$ y en grandes $(24,3 \%)$, en parte debido a las características propias del sistema de medios de la provincia de Málaga, donde son escasas las entidades de más de 100 empleados. En todos los grupos había más mujeres que hombres, con la proporción siempre entre el 60 y el $70 \%$, tal y como ocurre en las aulas del grado. La edad media también era similar en todas las compañías, en torno a los 23 años, si bien en las grandes existía una desviación típica algo menor.

Sí se da alguna pequeña diferencia en lo relativo al nivel de estudios. En apariencia, cuanto mayor es la empresa más frecuente es encontrar a estudiantes de

Revista de Comunicación de la SEECI. 2021, no 54, 79-95 
Máster $-1,5 \%$ de incidencias en las de pequeño tamaño frente a $8,6 \%$ en las grandes, casi seis veces más-, aunque constituyen en cualquier caso un colectivo minoritario. En cuanto a las áreas de especialización, en los medios pequeños se había asignado una sección específica al 90,9\% de la muestra, pero esta cifra descendía a medida que crecía la empresa, hasta el $81,4 \%$ en las medianas y hasta el $62,9 \%$ en las grandes -esto es, en ellas más de una tercera parte de los alumnos rotaba por las diferentes secciones-. También resulta llamativa la variabilidad en lo concerniente al período de la actividad: las entidades grandes reciben a más estudiantes durante la época estival que el resto del año - solo el $17,1 \%$ se incorpora entre octubre y junio-, mientras que en las demás se encuentran algo más repartidos -la cifra asciende al $27,8 \%$ en las pequeñas y al $34,9 \%$ en las medianas-. En cualquier caso, las prácticas de verano son las más extendidas con independencia de la magnitud de la institución de acogida.

Todos estos datos se detallan pormenorizadamente en la tabla 1.

Tabla 1. Datos de registro de la muestra en función del tamaño del medio.

\begin{tabular}{|cc|c|c|c|}
\hline & & Pequeñas (1-20) & Medianas (21-99) & Grandes (100+) \\
\hline $\mathrm{N}$ & & $45,8 \%$ & $29,9 \%$ & $24,3 \%$ \\
\hline Universidad & (UMA) & $34,8 \%$ & $32,6 \%$ & $5,7 \%$ \\
\hline Género & (Mujer) & $60,6 \%$ & $67,4 \%$ & $65,7 \%$ \\
\hline Edad & Media (DT) & $23,0(1,94)$ & $23,3(2,70)$ & $22,7(1,35)$ \\
& Mín. / Máx. & $20 / 32$ & $20 / 34$ & $21 / 27$ \\
\hline & Tercero & $25,8 \%$ & $16,3 \%$ & $22,9 \%$ \\
\multirow{4}{*}{ Nivel de } & Cuarto & $42,4 \%$ & $48,8 \%$ & $40,0 \%$ \\
estudios & Créditos sueltos & $18,2 \%$ & $20,9 \%$ & $14,3 \%$ \\
& Finalizados & $12,1 \%$ & $9,3 \%$ & $4,3 \%$ \\
& Máster & $1,5 \%$ & $4,7 \%$ & $8,6 \%$ \\
\hline Sección & (Itinerante) & $9,1 \%$ & $18,6 \%$ & $37,1 \%$ \\
\hline Período & (Verano) & $71,2 \%$ & $65,1 \%$ & $82,9 \%$ \\
\hline
\end{tabular}

Fuente: Elaboración propia a partir de las encuestas a estudiantes en prácticas.

\subsection{Condiciones y rutinas de las prácticas}

La remuneración promedio recibida por los estudiantes es similar en todas las entidades contempladas -entre los 280,81 euros de las medianas y los 296,94 de las grandes-, pero las diferencias en las desviaciones típicas (ver tabla 2) exigen un análisis más pausado.

Tabla 2. Condiciones de las prácticas en función del tamaño del medio. 
García-Borrego, M., Gómez-Calderón, B. y Córdoba-Cabús, A Menos no siempre es más. Cómo el tamaño de los medios condiciona las prácticas extracurriculares en Periodismo

\begin{tabular}{|c|c|c|c|}
\hline Remu & $295,67 €(164,96 €)$ & $280,81 €(126,82 €)$ & $296,94 €(94,07 €)$ \\
\hline Hora & $5 \mathrm{~h} 54 \mathrm{~m}$ ( & $6 \mathrm{~h} 13 \mathrm{~m}$ ( & $2 m)$ \\
\hline Piezas realizadas a & $4,82(3,65)$ & $3,98(2,61)$ & $4,15(4,02)$ \\
\hline
\end{tabular}

Fuente: Elaboración propia a partir de las encuestas a estudiantes en prácticas.

Así, se observan distintos patrones en lo relativo a la bolsa de ayuda concedida a los alumnos: es más común que no reciban prestación de ningún tipo en las redacciones pequeñas y medianas -es el caso del $13,7 \%$ y $10,4 \%$ de los encuestados, respectivamente-, mientras que en las grandes este grupo apenas representa el 2,7\% del total. Sin embargo, una vez se traspasan ciertos umbrales se manifiesta el fenómeno contrario: son las más pequeñas las que suelen desembolsar mayores cantidades a la hora de bonificar a sus alumnos. En este sentido, destaca que más de la mitad de los estudiantes en medios pequeños $(51,5 \%)$ sobrepasa la cifra de 300 euros frente al $38,1 \%$ de los medianos y el $22,9 \%$ de los grandes. Esta peculiaridad en las remuneraciones queda reflejada en el gráfico 1.

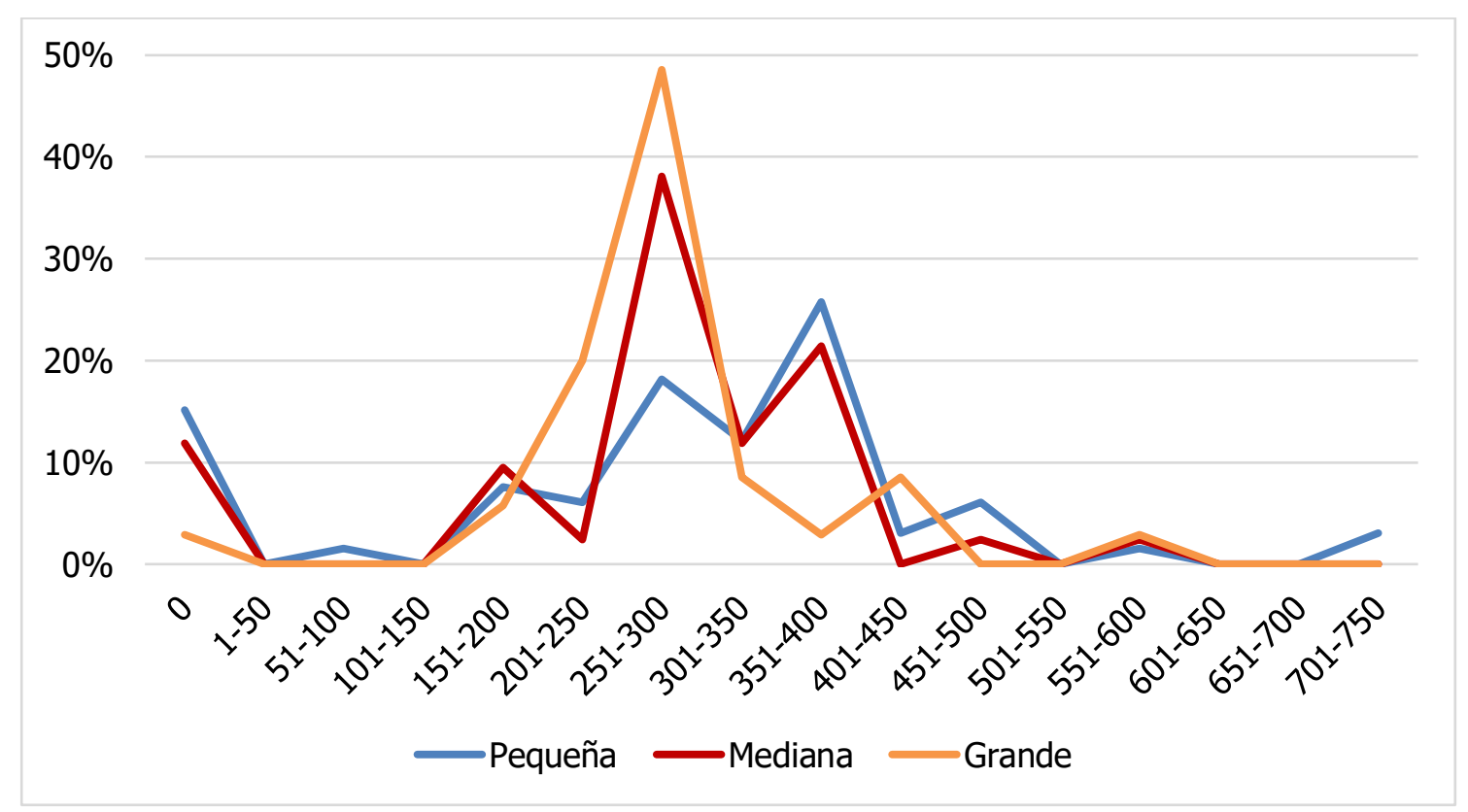

Gráfico 1: Distribución de la remuneración según el tamaño de la empresa.

Fuente: Elaboración propia a partir de las encuestas a estudiantes en prácticas.

En los horarios sí se percibe cierto escalonamiento, de manera que el tránsito de un medio menor a otro mayor acarrea un incremento de la jornada de aproximadamente 30 minutos. De esta manera, los estudiantes en empresas pequeñas trabajarían en torno a una hora menos de promedio que los de las grandes ( 5 horas y 54 minutos frente a 6 horas y 44 minutos). De nuevo esta distribución no es homogénea, aunque el patrón en este caso resulta algo más evidente: en entidades pequeñas solo rebasan las 6 horas el 26,5\% de los alumnos; en medianas, el $37,2 \% ; y$, en grandes, el 58,7\%. Estos datos casan con el tipo de jornada de los estudiantes: en las pequeñas el $45,5 \%$ disfruta de turno solo de mañana, un tipo de 
García-Borrego, M., Gómez-Calderón, B. y Córdoba-Cabús, A Menos no siempre es más. Cómo el tamaño de los medios condiciona las prácticas extracurriculares en Periodismo

horario bastante menos habitual en el resto de instituciones ( $23,3 \%$ en medianas y $28,6 \%$ en grandes).

En lo tocante al número de piezas realizadas por los encuestados, son los de medios pequeños los que arrojan un mayor promedio (4,82 por día, frente a 3,98 de los medianos y 4,15 de grandes). Esto se debe, principalmente, a que entre ellos se encuentra el grupo estimable de alumnos $(13,8 \%)$ que realizan 10 textos o más, ya que si se sitúa la referencia en 5 piezas, las cifras son coincidentes -en torno a una cuarta parte de los estudiantes supera esta carga en cualquier redacción-.

El cuestionario también indagaba sobre el grado de proactividad de los alumnos y la frecuencia con que afrontaban determinados dilemas éticos. Con respecto al primer asunto, existe cierta uniformidad en cuanto a proponer temas -lo hacen muy frecuentemente en el $65,1 \%$ de instituciones medianas y el $73,9 \%$ de pequeñas ${ }^{4}-$, pero se advierte gradación en lo referido al enfoque informativo: en entidades pequeñas suele intervenir en él -0 , cuando menos, se le permite manifestar su opinión- el $65,1 \%$ de los estudiantes. En medianas esta proporción cae al 58,2\%, y en grandes, al $51,4 \%{ }^{5}$.

Esta misma progresión, aunque a la inversa, se observa al preguntar a los alumnos si han firmado piezas que les desagradasen por haber sufrido cambios sustanciales con respecto al original. En los medios pequeños le ocurre habitualmente o siempre al $18,2 \%$ de los estudiantes, por el $28,0 \%$ de los medianos y el $31,4 \%$ de los grandes. Pese a que esto, a priori, hable en detrimento de las instituciones de mayor envergadura, conviene reseñar que es también en ellas donde se da una mayor proporción de encuestados que nunca ha afrontado este tipo de situación ( $51,4 \%$ del total), frente al $39,4 \%$ de las pequeñas y el $37,2 \%$ de las medianas. Por tanto, en los grandes medios es donde menos estudiantes firman piezas que les desagradan, pero también donde, cuando se produce esta coyuntura, lo hace con mayor intensidad. En cuanto a la realización de tareas contrarias a la ética, los resultados son parejos en todos los medios: aproximadamente la mitad de los consultados las había llevado a cabo sin que resultara de importancia el tamaño de la empresa.

Por último, se quiso saber en qué medida difería el contacto de los alumnos con los dos tutores, el profesional, designado por la empresa, y el académico, representante de la universidad ${ }^{6}$. La preponderancia del profesional es evidente en todos los casos, pero a medida que aumenta el tamaño de la redacción, la relación pierde fluidez. En los medios pequeños, el $31,8 \%$ dirime sus asuntos con esta figura

${ }^{4}$ Cifra obtenida de la suma de las opciones "Siempre" y "Habitualmente", presentada así para facilitar la lectura de los resultados.

${ }^{5}$ De nuevo, el dato procede de la suma de las opciones "Siempre" y "Habitualmente".

${ }^{6}$ El Real Decreto 592/2014, de 11 de julio, prevé la existencia de dos tutores, uno de la entidad colaboradora y otro de la universidad, que se deben responsabilizar de supervisar y guiar las actividades del estudiante, hacer un "seguimiento efectivo" de las prácticas y, en definitiva, "velar por el normal desarrollo del Proyecto Formativo".

Revista de Comunicación de la SEECI. 2021, n 54, 79-95 
a diario, y un 10,6\% adicional lo hace varias veces a la semana (en total, $42,4 \%$ ); en los grandes, los porcentajes descienden respectivamente hasta un 17,1 y un $5,7 \%$ (sumados, un $22,8 \%$ de la muestra). De hecho, en las redacciones grandes son mayoría quienes no conocen o no han trabado contacto nunca con su tutor profesional $(51,5 \%)$, mientras que en las pequeñas la proporción, aun siendo también alta, no pasa de un tercio $(33,4 \%)$. El tutor académico, en cambio, es desconocido por prácticamente la mitad de los estudiantes, y apenas un $7,6 \%$ se ha relacionado con él en las empresas pequeñas, un $2,3 \%$ en las medianas y un $2,9 \%$ en las grandes (ver gráficos 2 y 3 ).

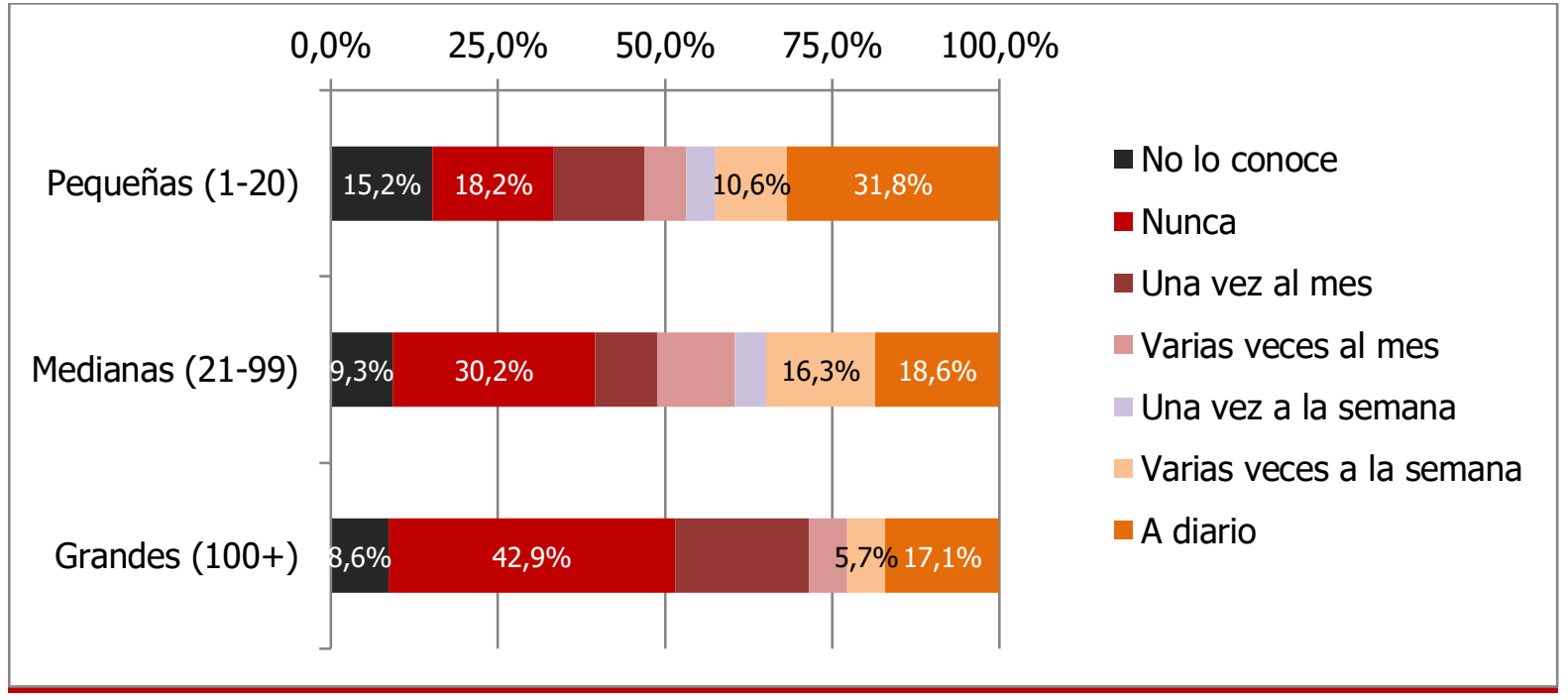

Gráfico 2: Frecuencia del contacto con el tutor de empresa.

Fuente: Elaboración propia a partir de las encuestas a estudiantes en prácticas.

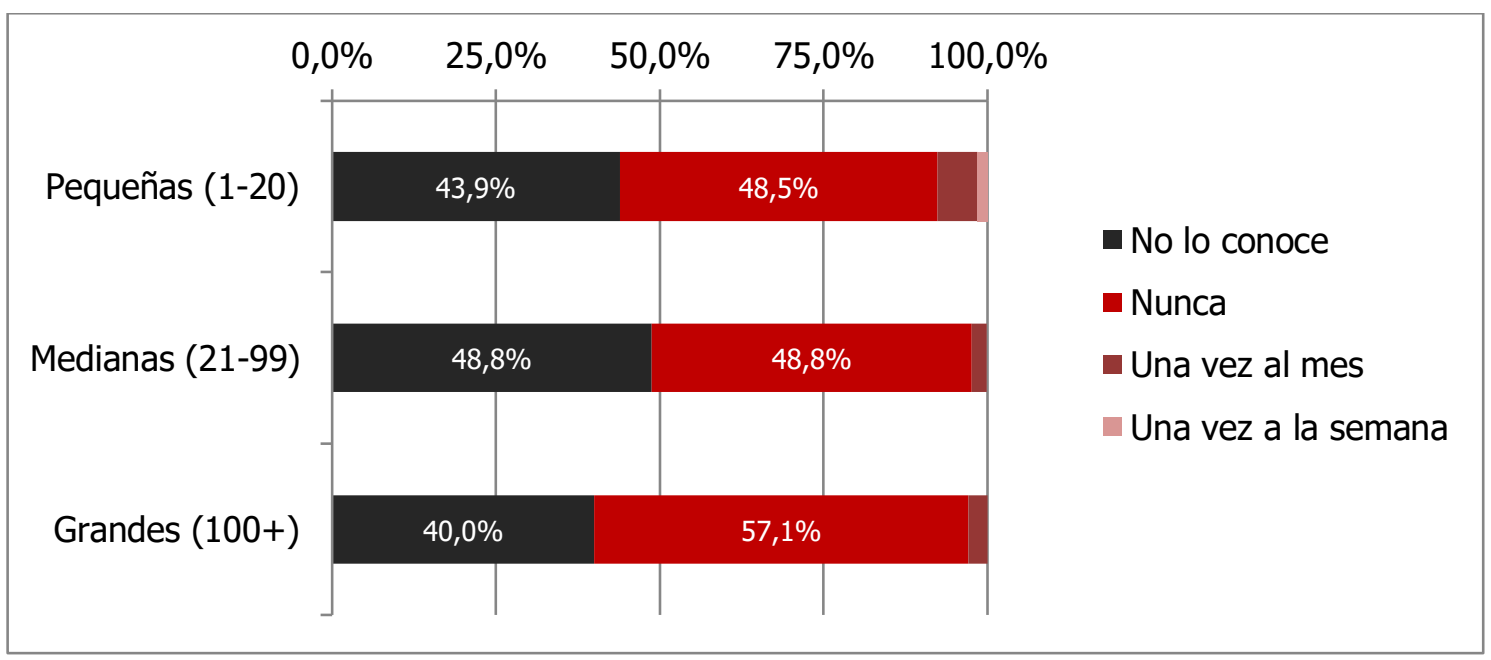

Gráfico 3: Frecuencia del contacto con el tutor de la universidad.

Fuente: Elaboración propia a partir de las encuestas a estudiantes en prácticas. 
García-Borrego, M., Gómez-Calderón, B. y Córdoba-Cabús, A Menos no siempre es más. Cómo el tamaño de los medios condiciona las prácticas extracurriculares en Periodismo

\subsection{Niveles de satisfacción}

El listado de 11 variables que se emplearon para medir la satisfacción de los estudiantes con las prácticas no arroja diferencias estadísticamente significativas al discriminar en función del tamaño de la empresa de acogida. Sí se aprecian leves patrones coincidentes con las condiciones y rutinas previamente detalladas -la insatisfacción más pronunciada en los estudiantes de medios grandes con el tutor de empresa, con quien apenas mantienen contacto; la de los alumnos de instituciones medianas con la remuneración, más baja en promedio que la del resto de compañeros; o la satisfacción menguante con los horarios a medida que crece el tamaño de la empresa, en línea con el número de horas dedicadas-, pero las diferencias apenas son apreciables, como se puede comprobar en la tabla 3.

Tabla 3. Satisfacción con las prácticas en función del tamaño del medio.

\begin{tabular}{|c|c|c|c|}
\hline & Pequeño (1-20) & $\begin{array}{l}\text { Mediano (21- } \\
\text { 99) }\end{array}$ & Grande $(100+)$ \\
\hline Satisfacción con tutor de empresa & $2,88(\mathrm{DT}=1,43)$ & $2,95(\mathrm{DT}=1,54)$ & $2,51(\mathrm{DT}=1,52)$ \\
\hline Satisfacción con tutor académico & $1,68(\mathrm{DT}=1,09)$ & $1,79(\mathrm{DT}=1,19)$ & $1,51(\mathrm{DT}=0,74)$ \\
\hline $\begin{array}{c}\text { Satisfacción con trabajadores de la } \\
\text { empresa }\end{array}$ & $4,08(\mathrm{DT}=1,21)$ & $3,81(\mathrm{DT}=1,14)$ & $4,14(\mathrm{DT}=1,12)$ \\
\hline Satisfacción con otros becarios & $3,88(\mathrm{DT}=1,38)$ & $3,79(\mathrm{DT}=1,37)$ & $4,23(\mathrm{DT}=1,06)$ \\
\hline Satisfacción con superiores & $3,35(\mathrm{DT}=1,43)$ & $3,09(\mathrm{DT}=1,23)$ & $3,37(\mathrm{DT}=1,35)$ \\
\hline Satisfacción con horarios & $3,41(\mathrm{DT}=1,34)$ & $3,19(\mathrm{DT}=1,20)$ & $2,97(\mathrm{DT}=1,34)$ \\
\hline Satisfacción con estabilidad de la beca & $2,77(\mathrm{DT}=1,56)$ & $2,42(\mathrm{DT}=1,24)$ & $2,54(\mathrm{DT}=1,50)$ \\
\hline Satisfacción con carga de trabajo & $3,12(\mathrm{DT}=1,31)$ & $2,86(\mathrm{DT}=1,28)$ & $3,26(\mathrm{DT}=1,22)$ \\
\hline Satisfacción con línea editorial & $2,78(\mathrm{DT}=1,44)$ & $2,69(\mathrm{DT}=1,07)$ & $2,62(\mathrm{DT}=1,05)$ \\
\hline $\begin{array}{l}\text { Satisfacción con oportunidades de } \\
\text { promocionar }\end{array}$ & $2,36(\mathrm{DT}=1,43)$ & $2,30(\mathrm{DT}=1,32)$ & $2,14(\mathrm{DT}=1,17)$ \\
\hline Satisfacción con remuneración & $2,11(\mathrm{DT}=1,27)$ & $1,79(\mathrm{DT}=1,08)$ & $2,06(\mathrm{DT}=1,19)$ \\
\hline Promedio satisfacción & $2,95(D T=0,92)$ & $2,79(\mathrm{DT}=0,79)$ & $2,85(\mathrm{DT}=0,79)$ \\
\hline
\end{tabular}

Fuente: Elaboración propia a partir de las encuestas a estudiantes en prácticas.

Sin embargo, aun estando los estudiantes casi igual de satisfechos en todos los aspectos contemplados, las aspiraciones de futuro sí difieren notablemente según la envergadura de la empresa en la que se encuentran. En los medios pequeños y medianos son mayoría quienes, en un plazo de cinco años, querrían cambiar a una redacción distinta $(50,0 \%$ y $51,2 \%$, respectivamente) y minoría los que desearían continuar $(16,7 \%$ y $14,0 \%)$, superados incluso por quienes no tienen claras sus preferencias $(18,2 \%$ y $30,2 \%)$. En empresas grandes se da el fenómeno contrario: abundan los que querrían permanecer en ellas $(42,9 \%)$, aunque también un porcentaje nada desdeñable optaría por cambiar de medio si le fuera posible $(28,6 \%)$ o manifiesta su incertidumbre al respecto $(20,0 \%)$. El salto a gabinetes o la posibilidad de abandonar el sector de la comunicación no parece entrar en los planes inmediatos de la mayoría de los encuestados, aunque esta última opción goza de mayor predicamento entre los alumnos de medios pequeños (ver tabla 4). 
García-Borrego, M., Gómez-Calderón, B. y Córdoba-Cabús, A Menos no siempre es más. Cómo el tamaño de los medios condiciona las prácticas extracurriculares en Periodismo

Tabla 4. Título de la tabla.

\begin{tabular}{c|c|c|c|} 
& Pequeña (1-20) & Mediana (21-99) & Grande (100+) \\
\hline Quiere quedarse en su medio & $16,7 \%$ & $14,0 \%$ & $42,9 \%$ \\
\hline Quiere cambiar a otro medio & $50,0 \%$ & $51,2 \%$ & $28,6 \%$ \\
\hline Quiere cambiar a un gabinete & $6,1 \%$ & $4,7 \%$ & $5,7 \%$ \\
\hline No lo tiene claro & $18,2 \%$ & $30,2 \%$ & $20,0 \%$ \\
\hline Quiere dejar el campo de la comunicación & $9,1 \%$ & $0,0 \%$ & $2,9 \%$ \\
\hline
\end{tabular}

Fuente: Elaboración propia a partir de las encuestas a estudiantes en prácticas.

\section{DISCUSIÓN}

El objetivo principal de esta investigación consistía en determinar las características de las prácticas extracurriculares en función del tamaño de la empresa en la que se realizan. Si bien es cierto que no se han hallado modelos radicalmente distintos a tenor de los datos expuestos, resulta factible plantear algunos rasgos diferenciales y ampliar de esta manera el conocimiento disponible sobre cómo se produce la primera toma de contacto de los estudiantes de Periodismo con el mercado laboral. El propósito final de los autores sería orientar sobre algunos aspectos que deberían corregirse en los actuales convenios universidad-empresa, para asegurar una experiencia de prácticas fructífera y garantista, algo que la literatura disponible ha echado en falta en los últimos veinte años, con las consecuencias que ello tiene para el ejercicio de la profesión (vid. Cantalapiedra et al., 2000; Blanco, 2005; Lamuedra, 2007; García-Borrego et al., 2017).

En líneas generales, en las empresas pequeñas, las prácticas se distinguen por estar más repartidas a lo largo del año, quizá porque los estudiantes son vistos como un refuerzo continuo para las plantillas, aunque sigue siendo en verano donde se concentra la mayor parte de los alumnos, normalmente en secciones fijas. Un número importante de estas prácticas no comporta remuneración, pero en aquellas instituciones en las que se compensa económicamente a los alumnos, la cuantía tiende a superar la de las empresas medianas y grandes. Las jornadas suelen ser más reducidas pero traen consigo una carga de trabajo equiparable a la del resto de medios, los estudiantes gozan de más libertad cuando se trata de decidir el enfoque - 0 , cuando menos, se tiene en consideración su opinión-, son minoría los que firman habitualmente noticias que les incomodan por haber sufrido cambios aunque buena parte de ellos lo ha hecho alguna vez- y mantienen una comunicación con los tutores, en particular con el profesional, más fluida que en el resto de empresas. Aun así, los índices de satisfacción se sitúan al mismo nivel que en los otros grupos, y son, de hecho, mayoría los que preferirían marcharse a otro medio si surgiera la posibilidad.

Las empresas medianas también presentan algunas características distintivas. Son las que mayor regularidad exhiben a la hora de convocar prácticas -aunque de nuevo se observa una clara preferencia por el periodo estival- y las que menos carga de trabajo implican para el estudiante, pero también las peor remuneradas, 
García-Borrego, M., Gómez-Calderón, B. y Córdoba-Cabús, A Menos no siempre es más. Cómo el tamaño de los medios condiciona las prácticas extracurriculares en Periodismo

tanto por existir un número estimable de puestos no retribuidos como por abonar cantidades más bien modestas en caso de gratificación. En el resto de aspectos contemplados - número de horas dedicadas, rotación por secciones, contacto con los tutores, libertad para intervenir en el enfoque y cambios sufridos por las piezas- se sitúan a medio camino entre las empresas pequeñas y las grandes. Sea por estos o por otros motivos, también son aquí mayoría los encuestados que manifiestan su deseo de dar el salto a otro medio en el futuro.

Por último, las empresas grandes destacan por la predominancia de las prácticas de verano - más del $80 \%$ de los casos-, tal vez porque, como denuncia la literatura científica, en buena medida los estudiantes cubren las ausencias de redactores sénior. Algo más cualificados que el resto de sus compañeros, también es más frecuente que los alumnos roten por las distintas secciones del medio en lugar de encuadrarse en un área fija. Casi la totalidad de las prácticas son remuneradas, aunque menos generosamente que en los medios pequeños y medianos, y suelen comportar una dedicación horaria mayor. En las rutinas se observa un mayor control por parte de la redacción - la influencia de los estudiantes en el enfoque noticioso es más limitada y buena parte de ellos sufre continuamente en sus artículos cambios con los que no está conforme-, aunque dicho control no lo ejerce el tutor, con quien mantienen la relación menos fluida de los tres modelos. Pese a todo, el sentimiento de pertenencia es mucho mayor, en tanto que, al contrario que los encuestados del resto de medios, en los grandes se da predomina la aspiración a permanecer en la empresa.

Estas constataciones deberían complementarse en futuros estudios atendiendo a determinadas variables que, si bien han sido contempladas, requieren de un análisis más profundo, probablemente a través de metodologías cualitativas. En este sentido, surgen cuestiones en torno al tipo de piezas realizadas, cuyas cifras resultan en determinadas circunstancias difíciles de explicar: ¿̇asta qué punto puede realmente un alumno en prácticas rebasar los diez textos diarios con jornadas de entre cinco y seis horas? ¿Se trata, en este caso, del mismo tipo de piezas que las de quienes realizan dos o tres, o por el contrario, cuentan con un menor grado de elaboración e implican esfuerzos menos intensos?

Planean también, en esta línea, cuestiones relativas al grado de aprendizaje extraído en cada uno de los medios, particularmente en función de las rutinas aplicadas. ¿Perciben los estudiantes que aprenden más cuantos más textos elaboran, fomentando así competencias como la rapidez y la eficacia, o se trata más bien de un trabajo mecánico -adaptación de notas de prensa, por ejemplo- y funcionan mejor las prácticas con una carga de trabajo focalizada en menos piezas?

De igual manera, se suscitan preguntas relativas a la satisfacción y las aspiraciones de los alumnos. En apariencia, las prácticas en empresas pequeñas se caracterizan por rutinas y condiciones más confortables: en la mayoría de los casos las jornadas son más reducidas, se recibe una remuneración más generosa, la opinión del estudiante es tenida más en cuenta, el contacto con los tutores es más fluido y solo rara vez se firman piezas que planteen problemas éticos. $Y$, sin

Revista de Comunicación de la SEECI. 2021, n 54, 79-95 
García-Borrego, M., Gómez-Calderón, B. y Córdoba-Cabús, A Menos no siempre es más. Cómo el tamaño de los medios condiciona las prácticas extracurriculares en Periodismo

embargo, la satisfacción es igual a la manifestada en medios de mayor tamaño: en última instancia, aunque imponen menos exigencias fuera de convenio y el trabajo en ellas es más gratificante, las empresas pequeñas no consiguen fidelizar más a sus alumnos en prácticas, antes al contrario. Esto puede deberse a una concepción romántica de la profesión, que lleva a los estudiantes a considerar que el mejor periodismo se desarrolla en las grandes corporaciones; a las oportunidades de promoción, que parecen más viables cuanto mayor es la envergadura del medio; o a factores relacionados con el aprendizaje u otros aspectos que han escapado al alcance de este estudio.

Sean cuales sean los motivos, los autores pretendíamos con nuestro trabajo profundizar en cómo determinan las entidades de acogida la experiencia de prácticas de los estudiantes de Periodismo, y en este sentido, creemos haber perfilado varios patrones diferenciadores. Sería deseable que los medios de mayor tamaño incorporaran algunas de las rutinas percibidas en las empresas pequeñas, donde el trato al estudiante parece más gratificante; y en cualquier caso, que todas las entidades antepusieran las necesidades formativas al criterio puramente mercantil.

\section{REFERENCIAS}

APM (2005-2018). Informe anual de la profesión periodística 2005-2018. Madrid: Asociación de la Prensa de Madrid.

Blanco, E. (2005). Mujer y poder en los medios: Dificultades para una incorporación plena. En APM, Informe anual de la profesión periodística 2005 (99-106). Madrid: Asociación de la Prensa de Madrid.

Cantalapiedra, M. J., Coca, C. y Bezunartea, O. (2000). La situación profesional y laboral de los periodistas vascos, en Zer. Revista de Estudios de Comunicación, 9, 169-182.

Cohen-Scali, V. (2003). The influence of family, social, and work socialization on the construction of the professional identity of young adults, en Journal of Career Development, 29 (4), 237-249.

Cotter, C. (2010). News talk. Investigating the language of Journalism. Cambridge: Cambridge University Press.

Dreyfus, H. L. y Dreyfus, S. E. (1986). Mind over machine. The power of human intuition and expertise in the era of the computer. Glasgow: Basil Blackwell.

García-Borrego, M. y Roses, S. (2016). Rutinas profesionales de los becarios de Periodismo: el caso de Málaga, en Zer. Revista de Estudios de Comunicación, 21 (41), 155-169. http://dx.doi.org/10.1387/zer.17267 
García-Borrego, M., Gómez-Calderón, B. y Córdoba-Cabús, A Menos no siempre es más. Cómo el tamaño de los medios condiciona las prácticas extracurriculares en Periodismo

García-Borrego, M., Roses, S. y Farias, P. (2017). Condiciones de las prácticas profesionales en medios de comunicación: un estudio empírico, en Revista Latina de Comunicación Social, 72, 430-452. http://dx.doi.org/10.4185/RLCS-2017-1173

Gollmitzer, M. (2014). Precariously employed watchdogs?, en Journalism Practice, 8 (6), 826-841. https://doi.org/10.1080/17512786.2014.882061

Gómez-Calderón, B., García-Borrego, M. y Fernández-Sande, M. (2019). Prácticas extracurriculares en el grado en Periodismo: rutinas profesionales, condicionantes y nivel de satisfacción de los alumnos, en El Profesional de la Información, 28 (6), 1-11. https://doi.org/10.3145/epi.2019.nov.10

Gravengaard, G. y Rimestad, L. (2014). Socializing journalist trainees in the newsroom: On how to capture the intangible parts of the process, en Nordicom Review, 35 (special issue), 81-95.

Hall, D. T. (1976). Careers in organizations. Pacific Palisades: Goodyear.

Jaakkola, M. (2019). Finland: Two academic paths into journalism-the dual model of journalism education. En E. Novak (Ed.), Accreditation and Assessment of Journalism Education in Europe: Quality Evaluation and Stakeholder Influence (1532). Baden-Baden: Nomos.

Lamuedra, M. (2007). Estudiantes de Periodismo y prácticas profesionales: el reto del aprendizaje, en Comunicar, 28, 203-211.

Lave, J. y Wenger, E. (1991). Situated learning: Legitimate peripheral participation. Cambridge: Cambridge University Press.

Pérez-Serrano, M. J., Rodríguez-Barba, D. y Rodríguez-Pallares, M. (2015). Mercado de la comunicación y estudiantes de Periodismo. Estructura de la demanda de perfiles profesionales, en Revista Latina de Comunicación Social, 70, 209-229. http://dx.doi.org/10.4185/RLCS-2015-1043

Real Decreto 592/2014, de 11 de julio, por el que se regulan las prácticas académicas externas de los estudiantes universitarios. https://bit.ly/2Tgn3JM

Splichal, S. y Sparks, C. (1994). Journalists for the 21st Century: tendencies of professionalization among first-year students in 22 countries. Nueva Jersey. Ablex Publishing.

Suárez-Villegas, J. C. (2015). Aspectos éticos y deontológicos de la actividad periodística online. Su percepción por los profesionales, en Revista Latina de Comunicación Social, 70, 91-109. http://dx.doi.org/10.4185/RLCS-2015-1036

Udías, C. (2006). El mercado de las prácticas, en Periodistas (FAPE), 5, 35-40. 
García-Borrego, M., Gómez-Calderón, B. y Córdoba-Cabús, A Menos no siempre es más. Cómo el tamaño de los medios condiciona las prácticas extracurriculares en Periodismo

UMA (2020). Guía de prácticas académicas externas en la Universidad de Málaga. https://bit.ly/32JX8gU

Túñez, M. (2009). Jóvenes y prensa en papel en la era Internet. Estudio de hábitos de lectura, criterios de jerarquía de noticias, satisfacción con los contenidos informativos y ausencias temáticas. Estudios sobre el Mensaje Periodístico, 15, 503-524.

Túñez, M. y Martínez, M. Y. (2014) Análisis del impacto de la función, las actitudes y las condiciones laborales del periodista en la producción de noticias: Hacia un periodismo de empresa, en Zer. Revista de Estudios de Comunicación, 19 (36), 169-182.

Wenger, E. (1998). Communities of Practice. Learning, meaning and identity. Cambridge: Cambridge University Press.

Weaver, D. H. (1998). The global journalist: News people around the world. Cresskill: Hampton Press.

Weaver, D. H. y Willnat, L. (Eds.) (2012). The global journalist in the 21st Century: News people around the world. Nueva York: Routledge.

Willig, I. (2016). We all think the same. Internships, craft and conservation. En: J. F. Hovden, G. Nygren y H. Zilliacus-Tikkanen (Eds.), Becoming a journalist. Journalism education in the Nordic countries (39-50). Gotemburgo: Nordicom.

\section{AUTORES:}

\section{Manuel García-Borrego}

Disfruta de un contrato posdoctoral en el Departamento de Periodismo de la Universidad de Málaga. En sus cinco años de actividad investigadora ha publicado más de una decena de artículos en revistas indexadas en Scopus como El Profesional de la información, Latina de Comunicación Social, Cuadernos.info o Estudios sobre el Mensaje Periodístico, así como capítulos de libro en editoriales como Tirant Lo Blanch Gedisa, Pirámide o Dykinson. También cuenta con una treintena de ponencias en congresos internacionales. Sus principales intereses de investigación son la formación en el grado en Periodismo y las prácticas externas curriculares y extracurriculares. manoletus@uma.es

Orcid ID: https://orcid.org/0000-0001-6207-8741

Google Scholar: https://scholar.google.es/citations?user=6MOjiLMAAAAJ\&hl=es

\section{Bernardo Gómez-Calderón}

Profesor Titular de Periodismo de la Universidad de Málaga, donde imparte docencia de Grado y Posgrado desde el año 1999. Investiga en las áreas de géneros periodísticos, información política y redes sociales, contando en su haber con más de 60 publicaciones científicas entre artículos, libros y capítulos de libro. Ha publicado en

Revista de Comunicación de la SEECI. 2021, nº 54, 79-95 
García-Borrego, M., Gómez-Calderón, B. y Córdoba-Cabús, A Menos no siempre es más. Cómo el tamaño de los medios condiciona las prácticas extracurriculares en Periodismo

las revistas Latina de Comunicación Social, Comunicar, Comunicación y Sociedad, Estudios sobre el Mensaje Periodístico y El Profesional de la Información, entre otras. Desde 2001, ha participado de forma ininterrumpida en proyectos de I+D nacionales y autonómicos. En la actualidad es coordinador del Máster en Investigación sobre Medios de Comunicación, Audiencias y Práctica Profesional en Europa bjgomez@uma.es

Orcid ID: https://orcid.org/0000-0003-2537-7042

Google Scholar: https://scholar.google.es/citations?user=QJV5oDYAAAAJ\&hl=es

\section{Alba Córdoba-Cabús}

Doctoranda con una Ayuda de Formación del Profesorado Universitario (FPU). Graduada en Periodismo por la Universidad de Málaga en 2016 y Máster en Investigación sobre Medios de Comunicación, Audiencias y Práctica Profesional en Europa en 2017. Durante sus estudios realizó prácticas en diversos medios y empresas de comunicación (El Día de Córdoba, Canal Málaga RTV y Coonic) y fue alumna colaboradora en el Departamento de Periodismo con una beca del Ministerio. Tras finalizar su formación trabajó como Técnico en Gestión y Organización de Eventos en el Ayuntamiento de Montilla y en el Departamento de Comunicación, Eventos y Exposiciones de Tragsatec. Sus principales trabajos se centran en Periodismo de Datos, identificando patrones y cómo se materializa la información. albacordoba@uma.es

Orcid ID: https://orcid.org/0000-0002-3519-0583

Google Scholar: https://scholar.google.es/citations?user=xzzMtQ0AAAAJ\&hl=es 